\title{
Una evaluación crítica de los programas alimentarios en Argentina
}

\author{
A critical evaluation of food programs in Argentina
}

${ }^{1}$ Médico pediatra. Magíster Scientificae en Nutrición con énfasis en Salud Pública Materno-Infantil. Coordinador del Área de Nutrición, Dirección Nacional de Maternidad e Infancia, Ministerio de Salud de la Nación, Buenos Aires, Argentina. $\square$ (iD
RESUMEN Argentina tiene una historia de más de 60 años de programas alimentarios con escasísimas o nulas evaluaciones, aun cuando se han producido cambios epidemiológicos y sociales muy importantes en su población objetivo. En este artículo se revisan los programas alimentarios del Estado de alcance nacional, que tengan como propósito de su formulación contribuir a la seguridad alimentaria de las familias más necesitadas; se actualiza la situación nutricional de su población objetivo; y se proponen argumentos para analizar los programas vigentes a partir de una tipología basada en los componentes de la seguridad alimentaria. Una revisión general de los programas alimentarios requiere ser realizada desde su concepción, contenido y alcance, para que dejen de ser meras ayudas sociales y se transformen en portadores del derecho a una mejor alimentación y nutrición.

PALABRAS CLAVES Programas y Políticas de Nutrición y Alimentación; Seguridad Alimentaria y Nutricional; Nutrición en Salud Pública; Argentina.

\begin{abstract}
Argentina has an over 60-year history of food programs which have received few to no evaluations, even considering the highly important epidemiological and social changes that have occurred in the target population. In this article, nationwide State-run food programs with the objective of contributing to the food security of the families in greatest need are reviewed, the nutritional situation of the target population is reassessed, and arguments for analyzing current programs using a typology based in the components of food security are proposed. A general review of the conceptions, content and reach of food programs should be carried out, in order for these programs to cease to act as mere social assistance and instead be transformed in bearers of the right to better food and nutrition.
\end{abstract}

KEY WORDS Nutrition Programs and Policies; Food and Nutrition Security; Nutrition, Public Health; Argentina. 


\section{INTRODUCCIÓN}

La satisfacción de las necesidades alimentarias de la población forma parte de las prioridades esenciales que en nuestra especie permitieron crear, desde la más remota antigüedad, organizaciones sociales muy complejas $^{(1)}$. Las necesidades alimentarias fueron satisfechas con diversas intervenciones apropiadas a cada contexto histórico y grupo social de las sociedades estatales preindustriales $^{(2)}$. En Argentina se registran antecedentes en 1823 con la creación de la Sociedad de Beneficencia para paliar las necesidades de los más necesitados, aunque de alcance limitado ${ }^{(3)}$.

El presente trabajo tiene como objetivo reflexionar sobre los programas alimentarios del Estado con alcance nacional que tienen como propósito de su formulación contribuir a la seguridad alimentaria de las familias más necesitadas y así poder formular propuestas para aproximar sus resultados a ese propósito. Estos programas son básicamente de tres tipos: a) programas de ayuda o asistencia alimentaria (food aid), realizados como donaciones de alimentos entre países; b) de emergencia o catástrofe, con recursos propios del país, o también como ayuda solidaria internacional; c) como suplemento del ingreso familiar en dinero que permite la compra de alimentos (por ejemplo, Asignación Universal por Hijo $(\mathrm{AUH})$ - o entrega directa de alimentos - por ejemplo, Programa Materno Infantil (PMI).

El concepto de seguridad alimentaria surge a mediados de la década de 1970 con énfasis en la producción y disponibilidad alimentaria a nivel global. En la década de 1980 se agrega la idea del acceso, tanto económico como físico, y en la de 1990 se llega al concepto actual que incorpora la inocuidad y las preferencias culturales, y se la reafirma como un derecho humano. Estas miradas cambiantes se han dirimido dentro del Comité de Seguridad Alimentaria Mundial ${ }^{(4)}$. Por otro lado, y por iniciativa del movimiento Vía Campesina, se amplía el concepto de "seguridad alimentaria" a "soberanía alimentaria":
La soberanía alimentaria es el derecho de los países y los pueblos a definir sus propias políticas agrarias, de empleo, pesqueras, alimentarias y de tierra de forma que sean ecológica, social, económica y culturalmente apropiadas para ellos y sus circunstancias únicas. Esto incluye el verdadero derecho a la alimentación y a producir los alimentos, lo que significa que todos los pueblos tienen el derecho a una alimentación sana, nutritiva y culturalmente apropiada, y a la capacidad para mantenerse a sí mismos y a sus sociedades. ${ }^{(5)}$

En Argentina -país excedentario en alimentos- la dificultad de los hogares de contar con seguridad alimentaria radica fundamentalmente en la accesibilidad a los alimentos. Es por ello que las iniciativas, fundamentalmente desde el Estado nacional, se han orientado a paliar esa situación de crisis de accesibilidad.

Por ello es que en este trabajo nos referiremos, fundamentalmente, a los programas e iniciativas que, desde el Estado nacional, están orientadas a proveer un suplemento al ingreso del hogar para contribuir a satisfacer la necesidad prioritaria de la alimentación, integrándose como parte de las políticas sociales del Estado ${ }^{(6)}$. El proceso analítico de las políticas sociales suele realizarse desde los aspectos organizativos, institucionales, de los procesos y sus resultados, dejando de lado el enfoque de los derechos en relación con esas políticas $^{(7)}$. Si bien nuestro enfoque aquí está orientado a mejorar la oferta que brindan los programas alimentarios en cuanto a los aspectos organizativos, institucionales y/o procesales, no desconocemos que lo prioritario es responder a la vulneración de derechos sociales que el Estado debe garantizar desde la propia organización y caracterización social de su población. Desde esta perspectiva, tanto los programas alimentarios, como las políticas sociales en general, deben contribuir a la plena realización de esos derechos económicos y sociales y no tan solo a satisfacer necesidades. El Estado, en cuanto expresión social de organización de la comunidad, 
debe promover y facilitar los procesos para que la propia alimentación de la población sea la culminación de los derechos económicos y sociales con sentido de equidad y no tan solo un mero proceso para aquietar el hambre ${ }^{(8)}$.

\section{ANTECEDENTES DE LOS PROGRAMAS ALIMENTARIOS}

La promulgación de la Ley 12341 en 1936, habitualmente denominada "Ley Palacios", es el primer intento serio de dar respuesta desde el Estado a los problemas de salud, alimentación y nutrición de la población materno-infantil de Argentina, en situación de pobreza, con características de formulación similares a las de otros países enmarcados en la organización del Estado de bienestar ${ }^{(9)}$. Esta Ley crea y organiza la Dirección de Maternidad e Infancia dependiente, por ese entonces, del Ministerio del Interior. La Ley fue reglamentada en 1938 mediante el Decreto 5520, luego de resolver conflictos legales con el Patronato Nacional de Menores, dependiente del Ministerio de Justicia e Instrucción Pública, instancia que se había hecho cargo del bienestar de los niños hasta ese momento. Por dificultades operativas y de competencias jurisdiccionales, la Dirección de Maternidad e Infancia tuvo escasa presencia por fuera del centro metropolitano por lo que, en 1946, con la llegada de Perón al poder y como parte de una serie de modificaciones institucionales, se crea la Secretaría de Salud Pública, de la cual pasa a depender la Dirección. Recién en 1952, como parte de las intervenciones del Estado, se inicia la entrega regular de un alimento para mejorar la situación nutricional de la población más pobre. La entrega se realizaba en los centros materno-infantiles para una "distribución más lógica, racional y equitativa de la leche"(10) ya que el $70 \%$ de la producción láctea era consumida en la región conformada por las provincias de Buenos Aires, Santa Fe y Entre Ríos, en detrimento del resto del país. Con esta medida se preveía controlar la elevada mortalidad infantil, consecuencia, principalmente, de la desnutrición y de la diarrea estival(10).

Antes, en 1932, se había sancionado la Ley 11597, mediante la cual se proporcionaban fondos para el mantenimiento de los comedores escolares dependientes del Consejo Nacional de Educación y, en 1934, se había creado la Junta Nacional de Ayuda al Niño que tenía por función "socorrer al niño en edad escolar con alimentos y vestido"(11).

Luego de la Ley 12341 no hay registro de un presupuesto específico para programas alimentarios sino hasta 1973, a fines del gobierno de facto del teniente general Alejandro Agustín Lanusse, cuando se promulga la Ley 20445 que indica: "Asistencia alimentaria destinada a prevenir y tratar la desnutrición infantil y materna con la entrega de leche en polvo al $70 \%$ de los menores de cinco (5) años de los grupos más expuestos"(12). En esta Ley también se establece:

\begin{abstract}
Art. $3^{\circ}$. Para el ejercicio 1974 y sucesivos, el Poder Ejecutivo Nacional incorporará al presupuesto general de la Nación, dentro de las previsiones correspondientes a la Jurisdicción 60 - Ministerio de Bienestar Social (Subsecretaría de Salud Pública), una partida de cien millones de pesos (\$ 100.000.000), con la finalidad de asegurar en el futuro, los beneficios que se acuerdan por la presente ley. ${ }^{(12)}$
\end{abstract}

Desde entonces, se incorpora al Presupuesto Nacional una partida específica para sostener el Programa Materno Infantil y la compra de leche.

\section{PROGRAMAS ALIMENTARIOS DE LOS ÚLTIMOS 30 AÑOS}

Desde el advenimiento de la democracia, en 1983, hasta la fecha, hubo múltiples programas que respondían a diversos enfoques de formulación e implementación enmarcados en diferentes paradigmas. La promesa de campaña de Alfonsín de 1983 
"con la democracia se come..." se concretó en menos de seis meses con el lanzamiento, en marzo de 1984, del Plan Alimentario Nacional (PAN). En ese momento, el procesamiento de los datos del Censo Nacional de Población y Vivienda de 1980 permitió estimar la magnitud y distribución de la pobreza en el país ${ }^{(13)}$, la cual se había constituido en un problema de grandes proporciones que obligó al gobierno a darle respuesta so pena de poner en riesgo la continuidad de la democracia recién recuperada. "La concepción del PAN estuvo enmarcada por la concepción gubernamental en relación con el crecimiento económico y su correlato de bienestar social", dice Belmartino ${ }^{(14)}$.

La presidencia anticipada de Carlos Menem, motivada por la hiperinflación y la crisis de gobernabilidad en los últimos meses del gobierno anterior, se acompañó de una profunda crisis social con preocupación por saqueos y estallido social, particularmente, en el conurbano bonaerense.

La respuesta planeada desde el Ministerio de Salud y Acción Social por el ministro Julio Corzo y el secretario de Acción Social Rubén Cardozo fue mantener la estructura del Plan Alimentario Nacional y anticipar un sistema complementario de emergencia para cubrir las necesidades de los sectores más humildes ${ }^{(15)}$. Esta política asistencialista se expresó en el lanzamiento, en Rosario, ciudad de la provincia de Santa Fe, del Bono Nacional Solidario de Emergencia, más conocido como "Bono Solidario", creado por el Decreto 400 de 1989 "destinado a contribuir a la atención de las necesidades alimentarias y mínimas del sector más postergado de la población"(16). Este Decreto de Necesidad y Urgencia (DNU) designaba al Ministerio de Salud y Acción Social como la autoridad de aplicación y creaba el Consejo Nacional para la Emergencia Social presidido por el Ministerio de Salud y Acción Social e integrado por los Ministros del Interior y de Trabajo y Seguridad Social, representantes de las Fuerzas Armadas, de la Iglesia Católica, de la Confederación General del Trabajo (CGT) y de las organizaciones empresariales de la industria y el comercio. Asimismo, se constituyeron consejos provinciales similares. Lo insólito fue la participación de la CGT y organizaciones empresarias en un programa nacional, ya que la Iglesia católica, a través de Caritas y las Fuerzas Armadas, venían realizando acciones directas en situaciones de emergencia y actuando en la asistencia a los más necesitados.

Ante la necesidad de llegar de forma rápida a una alta proporción de familias necesitadas se pensó en la distribución de chequeras de bonos -con pocos controles (bastaba una declaración jurada de hallarse en estado de indigencia) - a través de autoridades políticas provinciales y municipales, diputados, senadores, parroquias, CGT y hasta punteros políticos de ciertas Unidades Básicas. El Bono Solidario preveía tres etapas: asistencia alimentaria a un millón de familias y grupos, autoabastecimiento familiar y microemprendimientos productivos. Solo se alcanzó la primera etapa, sumida en denuncias y acusaciones de corrupción desde la bancada opositora de la Cámara de Diputados porque "se ha gastado el $64 \%$ de la suma total en concepto de gastos de impresión, traslados y comisiones bancarias"(17). Sin embargo, seis meses después de su lanzamiento e implementación, Eduardo Bauzá, nuevo ministro por fallecimiento de Corzo, decía "si la gente busca alimentos, y se le da alimentos, no va a haber saqueos"(18).

El DNU de creación del Bono Solidario fue complementado con la Ley 23740 Programa de Emergencia Social que crea una contribución solidaria para su financiamiento con aportes importantes desde el sector privado. Esta inédita iniciativa no prosperó en la medida esperada y además el Bono Solidario fracasó por la manera discrecional de su distribución y lo patéticamente denigrante que fueron las largas colas en la CGT y su desviación hacia las dependencias del Ministro de Salud y Acción Social de la Nación cuando se agotaban las chequeras $^{(19)}$.

Las dificultades políticas y operativas de la asistencia social y del desarrollo humano, sumadas al convencimiento de la sociedad sobre la corrupción ministerial vinculada a 
la asistencia social ${ }^{(20,21)}$ Ilevaron, en 1999, a su escisión en dos ministerios, Salud por un lado y Desarrollo Social por el otro, que aún se mantiene.

En la década de 1990 predominó un conjunto de políticas neoliberales de reforma del Estado caracterizadas por la privatización, desregulación, liberalización comercial y financiera, y flexibilización del mercado de trabajo. Para limitar la conflictividad social, los programas sociales y, particularmente, los alimentarios se pensaron como políticas compensatorias y asistencialistas focalizadas en la población en situación de pobreza estructural y/o funcional. Las políticas neoliberales finalmente colapsaron y desencadenaron la crisis social, económica y política del 2001 con la renuncia del por entonces presidente Fernando De la Rua, bajo el lema "que se vayan todos", y la sucesión de cinco presidentes en menos de diez días. Luego de recuperada la institucionalidad, la etapa que transcurre entre 2003 y 2009 está marcada por la coordinación de las políticas sociales dirigidas a la seguridad alimentaria a través del Plan Nacional de Seguridad Alimentaria (PNSA). EI PNSA integra las diversas intervenciones vinculadas a la seguridad alimentaria con una coordinación única desde el Ministerio de Desarrollo Social que busca recuperar la comensalidad en el hogar y descentralizar las acciones hacia las provincias a través de la transferencia de recursos con el propósito de realizar intervenciones masivas en contraposición a la focalización. En 2009, con el lanzamiento del programa de Asignación Universal por Hijo $(\mathrm{AUH})$, comienza una nueva etapa marcada por la contribución del Estado a la plena realización de los derechos económicos y sociales de la población.

En la Tabla 1 se presentan los principales programas desde 1950 a 2015, identificados a partir de la modalidad operativa (tipología), la norma legal que le dio origen, el tiempo de vigencia y la población objetivo, y ordenados según año de inicio. El desarrollo temporal de estos programas se muestra en la Figura 1.

Luego de la crisis hiperinflacionaria de 1989, se multiplicaron las ollas populares y los comedores comunitarios, y el núcleo de las políticas alimentarias del Estado se sustentó en los programas de entrega de alimentos. Es decir, la década de 1990 se caracterizó por políticas públicas de focalización poblacional y el intento de la descentralización de la gestión para su distribución entre comedores comunitarios de diversos programas nacionales. Ejemplo de esto son el Programa Materno Infantil y Nutrición (PROMIN), el programa de Apoyo Solidario a los Mayores (ASOMA), el Programa Promoción del Bienestar de los Mayores (PPB), el Fondo Participativo de Inversión Social (FOPAR), el Programa Alimentario Nutricional Infantil (PRANI), y el programa Unidos ${ }^{(22)}$.

Con posterioridad a la crisis de 2001, en el marco de la emergencia alimentaria, se profundiza la descentralización de las políticas de intervención alimentaria, aunque se empieza a incorporar la educación alimentario-nutricional como herramienta para acompañar y hacer más efectiva la entrega de alimentos, como el Programa de Emergencia Alimentaria (PEA). En 2003, se promulga la Ley 25724 que da origen al Plan Nacional de Seguridad Alimentaria (PNSA), que continúa vigente e integra los componentes del programa de transferencia de fondos (como Educación Alimentaria y Nutricional; Abordaje Comunitario; ProHuerta) a través de tarjetas magnéticas, vales o tickets, cajas y bolsas de alimentos.

Con estos antecedentes, y con fuertes experiencias exitosas en toda la región, las intervenciones en políticas de seguridad alimentaria migraron en la mayoría de las provincias hacia la transferencia monetaria mediante tarjetas magnéticas, vales o tickets, en reemplazo de las cajas y bolsas de alimentos $\operatorname{secos}^{(23)}$. Tal es, por ejemplo, entre otros, el programa Vale Ciudad de la Ciudad Autónoma de Buenos Aires que se originó mediante la Ley 1506 de 2004, derogada en 2006 por la Ley 1878 de 2006 y el Vale Más de la provincia de Mendoza que fue la modalidad de aplicar las transferencias desde el PNSA. 
Tabla 1. Programas alimentarios implementados en Argentina, según tipología, norma legal, vigencia y población objetivo. 1950-2015.

\begin{tabular}{|c|c|c|c|c|}
\hline Nombre del programa & Tipología & Norma legal & Vigencia & Población objetivo \\
\hline $\begin{array}{l}\text { Programa Materno Infantil } \\
\text { (PMI) }\end{array}$ & Dación de alimentos & Ley 12341 & 1951-continúa & $\begin{array}{l}\text { Embarazadas, nodrizas y } \\
\text { niños demandantes del } \\
\text { subsector público }\end{array}$ \\
\hline $\begin{array}{l}\text { Programa de Promoción } \\
\text { Social Nutricional } \\
\text { (PROSONU) }\end{array}$ & $\begin{array}{l}\text { Dación de copa de } \\
\text { leche }\end{array}$ & Ley 24049 & 1972-1992 & Comedores escolares \\
\hline $\begin{array}{l}\text { Programa Alimentario } \\
\text { Nacional (PAN) }\end{array}$ & Dación de alimentos & Ley 23056 & 1984-1989 & $\begin{array}{l}\text { Familias en áreas con } \\
\text { necesidades básicas } \\
\text { insatisfechas }\end{array}$ \\
\hline Bono Solidario & $\begin{array}{l}\text { Otorga dinero a través } \\
\text { de tickets }\end{array}$ & $\begin{array}{l}\text { Decreto } 400 / 1989 \\
\text { Ley } 23740\end{array}$ & 1989-1990 & Familias pobres \\
\hline $\begin{array}{l}\text { Políticas Sociales } \\
\text { Comunitarias (POSOCO) }\end{array}$ & $\begin{array}{l}\text { Dación de alimentos a } \\
\text { través de comedores }\end{array}$ & Ley 23767 & 1989-2005 & Comedores comunitarios \\
\hline ProHuerta & $\begin{array}{l}\text { Autoproducción de } \\
\text { alimentos }\end{array}$ & $\begin{array}{l}\text { Resolución 239/1990 del } \\
\text { Instituto Nacional de Tecnología } \\
\text { Agropecuaria }\end{array}$ & 1990-continúa & Familias pobres rurales \\
\hline $\begin{array}{l}\text { Programa Promoción del } \\
\text { Bienestar de los Mayores } \\
\text { (PPB) }\end{array}$ & Dación de alimentos & $\begin{array}{l}\text { Resolución } 1517-\mathrm{D} / 1992 \text { del } \\
\text { Instituto Nacional de Servicios } \\
\text { Sociales para Jubilados y } \\
\text { Pensionados }\end{array}$ & 1992-continúa & $\begin{array}{l}\text { Afiliados al PAMI (Instituto } \\
\text { Nacional de Servicios } \\
\text { Sociales para Jubilados y } \\
\text { Pensionados) pobres }\end{array}$ \\
\hline $\begin{array}{l}\text { Programa Materno Infantil y } \\
\text { Nutrición (PROMIN) }\end{array}$ & Dación de alimentos & Decreto 443/1993 & 1993-2005 & $\begin{array}{l}\text { Embarazadas y niños } \\
\text { menores de } 6 \text { años en áreas } \\
\text { con necesidades básicas } \\
\text { insatisfechas }\end{array}$ \\
\hline $\begin{array}{l}\text { Apoyo Solidario a los } \\
\text { Mayores (ASOMA) }\end{array}$ & Dación de alimentos & Decreto $547 / 2000$ & 1994-2000 & $\begin{array}{l}\text { Afiliados al PAMI (Instituto } \\
\text { Nacional de Servicios } \\
\text { Sociales para Jubilados y } \\
\text { Pensionados) pobres }\end{array}$ \\
\hline $\begin{array}{l}\text { Programa Alimentario } \\
\text { Nutricional Infantil (PRANI) }\end{array}$ & $\begin{array}{l}\text { Dación de alimentos a } \\
\text { través de comedores }\end{array}$ & Decreto $547 / 2000$ & $1996-2000$ & $\begin{array}{l}\text { Niños mayores de } 2 \text { años de } \\
\text { familias pobres }\end{array}$ \\
\hline $\begin{array}{l}\text { Fondo Participativo de } \\
\text { Inversión Social (FOPAR) }\end{array}$ & $\begin{array}{l}\text { Dación de alimentos a } \\
\text { través de comedores }\end{array}$ & $\begin{array}{l}\text { Resolución 1826/1994 de la } \\
\text { Secretaría de Desarrollo Social }\end{array}$ & 1998-2005 & Comedores comunitarios \\
\hline Programa Unidos & Dación de alimentos & $\begin{array}{l}\text { Decreto } 547 / 2000 \\
\text { Decreto } 328 / 2002\end{array}$ & $2000-2002$ & Familias pobres \\
\hline Vale Más & $\begin{array}{l}\text { Otorga dinero a través } \\
\text { de tickets }\end{array}$ & $\begin{array}{l}\text { Fondos del Plan Nacional de } \\
\text { Seguridad Alimentaria }\end{array}$ & 2001-2008 & Familias pobres \\
\hline $\begin{array}{l}\text { Programa de Emergencia } \\
\text { Alimentaria (PEA) }\end{array}$ & Dación de alimentos & Decreto 328/2002 & $2002-2003$ & Familias pobres \\
\hline $\begin{array}{l}\text { Plan Nacional de Seguridad } \\
\text { Alimentaria (PNSA) }\end{array}$ & $\begin{array}{l}\text { Integra todos los tipos } \\
\text { anteriores }\end{array}$ & $\begin{array}{l}\text { Ley } 25724 \\
\text { Resolución } 2040 / 2003 \text { del } \\
\text { Ministerio de Desarrollo Social }\end{array}$ & 2003-continúa & Familias pobres \\
\hline $\begin{array}{l}\text { Asignación Universal por } \\
\text { Hijo (AUH) }\end{array}$ & $\begin{array}{l}\text { Otorga dinero a través } \\
\text { de transferencias }\end{array}$ & Decreto 1602/2009 & 2009-continúa & Niños \\
\hline $\begin{array}{l}\text { Asignación por Embarazo } \\
\text { para Protección Social }\end{array}$ & $\begin{array}{l}\text { Otorga dinero a través } \\
\text { de transferencias }\end{array}$ & Decreto 446/2011 & 2011-continúa & Embarazadas pobres \\
\hline $\begin{array}{l}\text { Programa Nacional de } \\
\text { Precios Cuidados }\end{array}$ & Acuerdo de precios & $\begin{array}{l}\text { Resolución } 20 / 2014 \text { de } \\
\text { la Secretaria de Comercio } \\
\text { apoyándose en la Ley } 24240 \\
\text { de Protección y Defensa del } \\
\text { Consumidor }\end{array}$ & 2014-continúa & Familias pobres \\
\hline
\end{tabular}




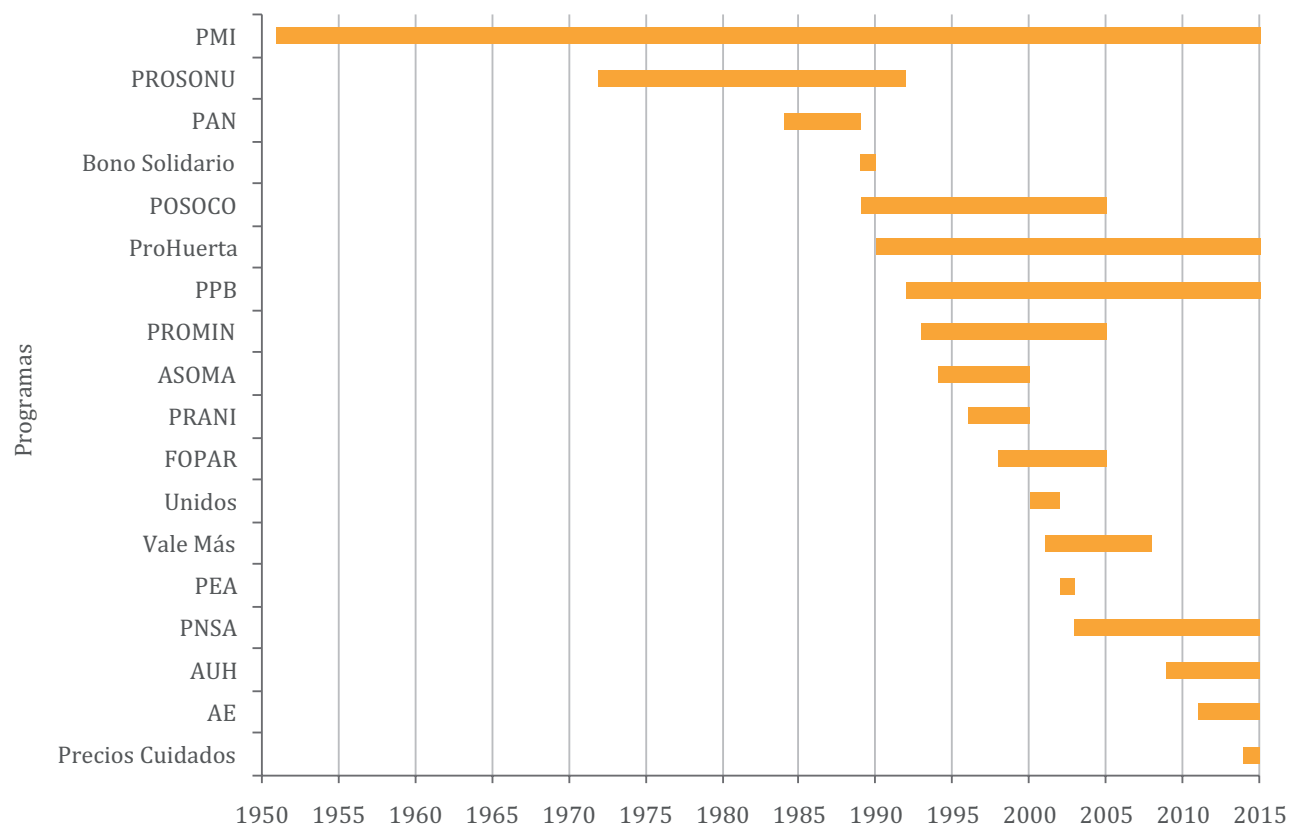

Figura 1. Programas alimentarios implementados en Argentina, según año de inicio. 1950-2015.

Fuente: Elaboración propia.

PMI = Programa Materno Infantil; PROSONU = Programa de Promoción Social Nutricional; PAN = Programa Alimentario Nacional; POSOCO = Políticas Sociales Comunitarias; PPB = Programa Promoción del Bienestar de los Mayores; PROMIN = Programa Materno Infantil y Nutrición; ASOMA = Apoyo Solidario a los Mayores; PRANI = Programa Alimentario Nutricional Infantil; FOPAR = Fondo Participativo de Inversión Social; PEA = Programa de Emergencia Alimentaria; PNSA = Plan Nacional de Seguridad Alimentaria; AUH = Asignación Universal por Hijo; AE = Asignación por Embarazo.

\section{PROGRAMAS ALIMENTARIOS ACTUALES}

\section{Programa Materno Infantil (PMI)}

Dentro de los componentes nutricionales del PMI, dependiente del Ministerio de Salud de la Nación, se encuentra la distribución de leche en polvo (desde 2001 fortificada con hierro y zinc según Ley 25459) a mujeres embarazadas y niños -a partir de los 6 meses y hasta los 6 años de edad-que se encuentren con compromiso nutricional. Además, se centra en educación, promoción de legislaciones y desarrollo de estrategias sanitarias para el mejoramiento de la situación nutricional de la población objetivo. Desde 2001, la distribución se realiza mediante una metodología consensuada por el Consejo Federal de Salud (organismo de consenso integral de políticas públicas de salud con una perspectiva federal) que integra fundamentalmente la cantidad de recién nacidos y un estimador de pobreza. De 1992 a 2003 se realizaron transferencias ${ }^{(24)}$ a cada una de las provincias para la compra de leche, cuyo monto total se incorporó a la Ley de Presupuesto Nacional y, desde 2003 hasta la actualidad, se realiza una compra semestral por licitación pública nacional que el adjudicatorio correspondiente entrega a cada una de las provincias. Los niveles de cobertura no han mantenido siempre niveles óptimos. En la Figura 2 se observa la evolución de la cobertura aparente de la leche entera entregada por el PMI como consolidado nacional. La 
cobertura aparente surge de considerar la población meta del PMI y la cantidad de leche comprada y entregada según las normas, con el presupuesto existente para el año considerado $^{(24)}$.

\section{Programa de Promoción Social Nutricional (PROSONU)}

Este programa depende parcialmente del Ministerio de Desarrollo Social y de las propias provincias. Su función esencial es el suministro de diferentes tipos de prestaciones (desayunos, meriendas, refrigerios, almuerzos) en escuelas públicas. Las provincias reciben transferencias financieras para adquirir alimentos.

\section{Programa Fondo Participativo de Inversión Social (FOPAR)}

Este programa creado en 1998 por la Resolución 1826/1994 de la Secretaría de Desarrollo Social opera a través de transferencias financieras y suministro de prestaciones para alimentación, mayoritariamente almuerzos, para espacios comunitarios con diversos grados de precariedad, en la modalidad de comedores comunitarios.

\section{ProHuerta}

Iniciativa implementada por el Instituto Nacional de Tecnología Agropecuaria (INTA) conjuntamente con el Ministerio de Desarrollo Social de la Nación. El objetivo es mejorar la seguridad alimentaria de la población urbana y rural en situación de vulnerabilidad social, incrementando la disponibilidad, accesibilidad y variedad de alimentos, así como la comercialización de excedentes, mediante la autoproducción de alimentos frescos que complementen sus necesidades alimentarias, en huertas y granjas con enfoque agroecológico, de acuerdo a las particularidades y costumbres de cada región. Se suministran semillas para autoproducción en unidades

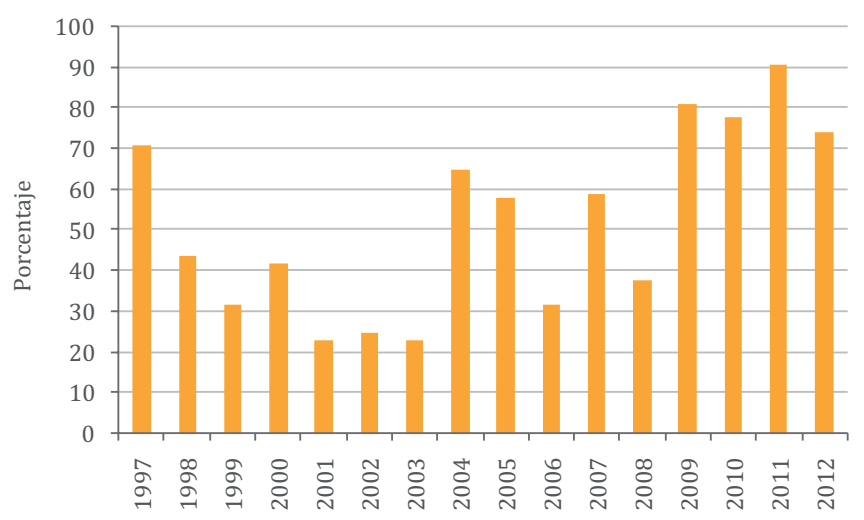

Figura 2. Porcentaje de cobertura aparente del Programa Materno Infantil. Argentina, 1997-2012.

Fuente: Elaboración propia a partir de datos de la Dirección Nacional de Maternidad e Infancia.

familiares, escolares o comunitarias a pequeña escala y se brinda capacitación a través de agentes agrícolas del INTA.

\section{Plan Nacional de Seguridad Alimentaria}

Inmediatamente después de la crisis de 2001, el Grupo Sophia en conjunto con Poder Ciudadano y Red Solidaria realizaron una propuesta técnica bajo el nombre "El hambre más urgente". El propósito era la atención de la nutrición, el cuidado de la salud y la estimulación temprana de todos los niños en situación de pobreza desde la concepción hasta los 5 años de edad en todo el país. Esta iniciativa fue acompañada por una campaña del periodista Luis Majul y el diario La Nación que permitió juntar más de un millón de firmas y lograr así el estatus de iniciativa popular para el consiguiente tratamiento parlamentario como lo indica el Artículo 39 de la Constitución de la Nación Argentina. Concomitantemente, en julio de 2002, el Consejo Nacional de Coordinación de Políticas Sociales realizó el Foro para un Plan Nacional de Alimentación y Nutrición con la participación de 300 técnicos de organismos gubernamentales, legisladores, investigadores de universidades y centros de 
investigación, y miembros de organizaciones no gubernamentales reunidos durante dos días en la Biblioteca Nacional ${ }^{(25)}$. Las conclusiones del Foro alimentaron la discusión parlamentaria y finalmente la propuesta original quedó plasmada en la Ley 25724 modificada y enriquecida ampliamente en este proceso y que fue promulgada de hecho en enero de 2003 por Kirchner. El rápido trámite parlamentario y su promulgación fueron un claro mensaje político sobre la prioridad puesta por el Gobierno en la "deuda social" frente a los requerimientos de la deuda financiera. La Ley 25724 crea el Programa Nacional de Nutrición y Alimentación, y en su Artículo $2^{\circ}$ define: "Dicho Programa en la emergencia, está destinado a cubrir los requisitos nutricionales de niños hasta los 14 años, embarazadas, discapacitados y ancianos desde los 70 años en situación de pobreza"(26). También especifica que los ministerios de Salud y de Desarrollo Social son las autoridades de aplicación de la ley y para la coordinación del programa crea la Comisión Nacional de Nutrición y Alimentación integrada por representantes del Ministerio de Salud, el Ministerio de Desarrollo Social y Medio Ambiente, el Ministerio de Educación, el Ministerio de Economía, el Ministerio de Trabajo, el Ministerio de Empleo y Formación de Recursos Humanos, el Ministerio de Producción, y de ONG debidamente acreditadas en el área. También comisiones provinciales y municipales similares. La operatividad quedó en la práctica a cargo del Ministerio de Desarrollo Social a través del Plan Nacional de Seguridad Alimentaria (PNSA). El propósito del PNSA es:

...financiar acciones de ejecución integral, tendientes a garantizar la Seguridad Alimentaria de personas en condición de vulnerabilidad social, priorizando la atención de la emergencia alimentaria [con el objetivo de] a) realizar un aporte a las necesidades alimentarias del hogar; b) fomentar mecanismos de asistencia y promoción que privilegien el ámbito familiar y el fortalecimiento de redes solidarias en la comunidad. ${ }^{(27)}$
Las prestaciones que este Plan financia son:

a) Asistencia Alimentaria a las familias;

b) Asistencia Alimentaria Directa; c) Asistencia en Comedores Escolares; d) Asistencia en Comedores Infantiles; e) Asistencia en Comedores de organizaciones de la sociedad civil; f) Asistencia Alimentaria para situaciones especiales, que hagan a pautas sociales y culturales; g) Asistencia para desnutridos y celíacos; h) Autoproducción de alimentos; i) Asistencia a huertas familiares; j) Asistencia a huertas comunitarias; k) Asistenciaahuertasescolares; I) Asistencia a granjas familiares; m) Asistencia a granjas comunitarias; n) Estimulación temprana y Desarrollo Infantil; o) Educación Alimentaria nutricional; p) Orientación en compras comunitarias; q) Asistencia Técnica y Capacitación; r) Formación de agentes de seguridad alimentaria (madres cuidadoras, agentes sanitarios, promotores sociales, maestros, jefes y jefas de hogar, estudiantes, profesionales, voluntarios, otros); s) Fortalecimiento de la gestión por medio de cooperación técnica a equipos provinciales y municipales; t) Equipamiento básico a efectores; u) Supervisión y Monitoreo de la ejecución del Plan; v) Evaluación del Estado nutricional de la población. ${ }^{(27)}$

\section{Asignación Universal por Hijo (AUH)}

Iniciado en 2009, este programa está enmarcado en un enfoque de política social desde una perspectiva de derechos pues incorpora a los hijos de familias de trabajadores sin acceso al Sistema de Protección Social ni al Régimen de Asignaciones Familiares. Es un programa de transferencia de ingresos que en circunstancias de pobreza se canalizan prioritariamente a la adquisición de alimentos.

Es un derecho que les corresponde a los hijos de las personas que están desocupadas, trabajan en la economía informal con ingresos iguales o inferiores al 
Salario Mínimo, Vital y Móvil, monotributistas sociales, trabajadores del servicio doméstico, trabajadores por temporada en el período de reserva del puesto o perciban alguno de los siguientes planes: Argentina Trabaja, Manos a la Obra, Ellas Hacen, Programa de Trabajo Autogestionado, Jóvenes con Más y Mejor Trabajo, Programa Promover la Igualdad de Oportunidades y Seguro de Capacitación y Empleo.

El cobro de la Asignación Universal por Hijo requiere la acreditación anual de escolarización y controles de salud de los niños. Se abona a los menores de 18 años, hasta un máximo de 5 hijos, priorizando a los hijos discapacitados y a los de menor edad. Se liquidará a uno solo de los padres priorizando a la mamá. ${ }^{(28)}$

La asignación es una transferencia directa del $80 \%$ y condicionada del $20 \%$ al cumplimiento de controles de salud y escolaridad. En 2013-2014 se realizó una evaluación del impacto de la $\mathrm{AUH}$ que, en lo sustantivo con relación a la alimentación, concluyó:

El ingreso complementario y estable que significa la $\mathrm{AUH}$ representa en promedio un cuarto del conjunto de ingresos familiares y para los hogares más pobres el 40 por ciento. Esto ha permitido asegurar la alimentación y un incremento en la cantidad y calidad de alimentos que consumen sus receptores. Se puede señalar al respecto que los acrecentamientos más sustanciales ocurren en rubros especialmente sensibles y prioritarios (proteínas, lácteos, frutas y verduras), indispensables para una nutrición saludable y equilibrada. ${ }^{(29)}$

Estas conclusiones dan por tierra los dichos de un encumbrado político opositor, quien afirmó: "La asignación universal por hijo se está yendo por la canaleta de la droga y el juego", lo cual expresa cabalmente el pensamiento retrógrado de buena parte de nuestra sociedad.

\section{Situación nutricional}

Con la Encuesta Nacional de Nutrición y Salud (ENNyS) ${ }^{(30)}$ realizada en 2004-2005 se tuvo conocimiento fehaciente de la distribución, características y magnitud de los problemas nutricionales del grupo maternoinfantil. Gracias a ese estudio se tiene evidencia de que los principales problemas en ese grupo poblacional son la anemia nutricional -fundamentalmente en los menores de 2 años $(41,1 \%$ de los niños de familias pobres y $29,4 \%$ de las no pobres) y mujeres embarazadas (30,5\%)-, obesidad (el 10,4\% de los niños menores de 6 años, el 15,6 $\%$ de las mujeres de 10 a 49 años con un máximo del $31 \%$ en las mayores) y baja talla en el $8,2 \%$ de los niños menores de 6 años. La proporción de estos problemas es más acentuada en las poblaciones pobres, indistintamente del área geográfica. También se identifican deficiencias dietéticas de calcio, vitaminas $\mathrm{A}, \mathrm{C}$ y $\mathrm{B}_{12}$ como se observa en la Tabla 2.

Esos déficits de nutrientes son el resultado de ingestas de dietas pobres en verduras, frutas, lácteos, aceites diferentes al girasol, legumbres y cereales integrales, al mismo tiempo que de un exceso de azúcares, bebidas azucaradas, carnes, y cereales muy refinados y de baja densidad de nutrientes.

Asimismo, los lactantes presentan un buen comienzo de la lactancia materna (95\%) pero solo el $45 \%$ y el $30 \%$ siguen con lactancia materna exclusiva al $4^{\circ}$ y $6^{\circ}$ mes, respectivamente ${ }^{(31)}$. Por otra parte, solo el $22 \%$ de los que no reciben lactancia materna en el segundo semestre y el $11 \%$ en el segundo año de vida, reciben alguna leche fortificada con hierro ${ }^{(32)}$.

La proporción de baja talla disminuyó un $45 \%$ en los menores de 5 años entre 2005 y 2013 como un consolidado nacional de los niños atendidos en centros de salud bajo la cobertura del Programa Sumar. Los mismos problemas nutricionales de baja talla y obesidad identificados por la ENNyS se presentan en niños de pueblos originarios de Chaco, Formosa, Misiones, Salta y Jujuy que demandan atención en los centros de salud ${ }^{(33)}$. 
Tabla 2. Porcentaje de niños, niñas y mujeres con ingesta inadecuada. Argentina, 2004-2005.

\begin{tabular}{|c|c|c|c|c|}
\hline Nutriente & $\begin{array}{l}\text { Niños y niñas de } \\
6 \text { a } 23 \text { meses }\end{array}$ & $\begin{array}{l}\text { Niños y niñas de } \\
2 \text { a } 5 \text { años }\end{array}$ & $\begin{array}{c}\text { Mujeres de } \\
10 \text { a } 49 \text { años }\end{array}$ & Embarazadas \\
\hline Energía & 13,7 & 29,1 & 57,8 & 64,3 \\
\hline Proteínas & 0,2 & 0,9 & 19,0 & 29,1 \\
\hline Ácidos grasos saturados & $\mathrm{N} / \mathrm{A}$ & 68,3 & 48,9 & 47,9 \\
\hline Ácidos grasos poliinsaturados & $\mathrm{N} / \mathrm{A}$ & 68,1 & 67,6 & 72,9 \\
\hline Hierro & 11,5 & 3,1 & 19,4 & 59,3 \\
\hline Calcio & 9,1 & 45,6 & 94,3 & 88,5 \\
\hline Vitamina A & 9,8 & 27,4 & 72,2 & 66,2 \\
\hline Vitamina C & 48,7 & 40,7 & 73,3 & 67,0 \\
\hline Zinc & 2,6 & 4,2 & 33,5 & 52,1 \\
\hline Folatos & 8,9 & 6,2 & 23,1 & 25,4 \\
\hline Vitamina $\mathrm{B}_{12}$ & 0,6 & 3,4 & 25,8 & 25,6 \\
\hline Colesterol & $\mathrm{N} / \mathrm{A}$ & 18,1 & 24,9 & 25,4 \\
\hline Fibra & $97,9 *$ & 97,8 & 97,2 & 93,8 \\
\hline \multicolumn{5}{|c|}{$\begin{array}{l}\text { Fuente: Elaboración propia a partir de datos de la Encuesta Nacional de Nutrición y Salud }{ }^{(30)} \text { y del Ministerio de Salud }{ }^{(32)} \text {. } \\
\text { N/A = No aplicable ya que no hay recomendaciones para este grupo etario. } \\
\text { aCorresponde al porcentaje de niños y niñas de } 6 \text { a } 23 \text { meses sin lactancia materna }{ }^{(32)} \text {. } \\
{ }^{*} \text { Estimado solo para niños y niñas de } 13 \text { a } 23 \text { meses por no haber recomendaciones para niños menores. }\end{array}$} \\
\hline
\end{tabular}

La obesidad es alarmante en los escolares, aumentando un 19,4\% entre 2007 y 2012 , como lo señala la encuesta en escolares ${ }^{(34)}$. Asimismo, esta tendencia se refleja en las encuestas nacionales de factores de riesgo realizadas en 2005, 2009 y 2013, aumentando $43,4 \%$ entre 2005 y 2013 , en los mayores de 18 años $^{(35)}$. Estos datos de encuestas, aunque con diferentes metodologías y en diferentes grupos de edad, señalan la importancia del sobrepeso y la obesidad en todos los grupos de edad, reflejo de la epidemia global de obesidad, como lo ha señalado la Organización Mundial de la Salud(36).

El análisis de la Encuesta de Gasto de los Hogares del Instituto Nacional de Estadística y Censos (INDEC) permite observar cómo los sectores de bajos ingresos han incorporado progresivamente, desde la década de 1990, hábitos alimentarios con dietas monótonas, y han perdido la diversidad de su canasta de alimentos, sustituyéndola por hidratos de carbono $^{(37)}$.

En síntesis, se puede decir que los principales problemas en función de su magnitud, extensión de grupos etarios afectados y trascendencia sanitaria son el sobrepeso y la obesidad, la anemia nutricional, la baja talla, los déficits de ingesta de nutrientes (como calcio y vitaminas $A, C$ y $B_{12}$ ) la baja práctica de la lactancia materna, y las altas ingestas de azúcares simples.

\section{LOS PROBLEMAS DE LOS PROGRAMAS ALIMENTARIOS ACTUALES}

El desarrollo de los programas alimentarios requiere prever en su formulación que en su implementación habrá diversos actores (funcionarios estatales, proveedores, beneficiarios) en diversos niveles jurisdiccionales (nacional, provincial/municipal, local) como de organización (operadores logísticos, equipo de salud, gestores sociales, efectores, etc) ${ }^{(38)}$. Esta complejidad sistémica sin una adecuada supervisión, monitoreo, seguimiento y retroalimentación, rápidamente se 
desarticula y se desvía del objetivo que le da origen. Una falencia generalizada de los programas sociales en general, y los alimentarios en particular, es la ausencia de monitoreo y evaluación. El monitoreo es parte de la propia gestión de los programas, provee información para reorientar las intervenciones con el fin de optimizar los procesos, los resultados y el impacto. Por otra parte, la evaluación está firmemente anclada en el conocimiento para la toma de decisiones sobre la continuidad o no de la intervención, de su ampliación o de su modificación. Es notable que, salvo muy escasas circunstancias, los programas no incorporen estos componentes y avancen a ciegas con los presupuestos preprogramáticos ${ }^{(39)}$.

Los programas de entrega de leche fortificada en América Latina han sido estrategias costo-efectivas para enfrentar las deficiencias de nutrientes, en particular, de hierro, como lo muestran las experiencias de México y Chile ${ }^{(40)}$ Sin embargo, las principales barreras que se identifican en Argentina para lograr el control de la anemia se deben, principalmente, a una falta de cobertura adecuada y la irregularidad en la entrega del beneficio a las familias, lo que facilita la dilución intrafamiliar hacia miembros que no necesariamente se benefician con la fortificación, en desmedro de los más necesitados. Por otra parte, la entrega de leche fortificada no se acompaña con educación a la familia en cuanto a su uso y mejor aprovechamiento. Otro tema que genera gran tensión en la entrega de leche fortificada, aunque no sea su propósito programático, es que en la práctica compite con la lactancia materna, tanto exclusiva, parcial como prolongada.

Hay un distanciamiento entre la naturaleza de la anemia y la propuesta programática. En Argentina, la anemia, como problema sanitario, es de origen nutricional y multifactorial, es decir que pueden confluir diversos factores como la ligadura precoz del cordón en el momento del nacimiento, la maternidad temprana de la adolescente, el breve intervalo intergenésico, la mala nutrición durante el embarazo, la incorporación temprana de semisólidos, la baja práctica de la lactancia materna exclusiva, el uso de alimentación complementaria inadecuada (baja densidad de nutrientes). La leche fortificada es solo un eslabón más en la multicausalidad, que tiene efecto al considerarla integralmente con los otros determinantes.

Los comedores escolares y comunitarios priorizan el rendimiento en términos de cantidad de raciones y no toman en cuenta la calidad de esas raciones. Las orientaciones dietéticas escasamente se cumplen. Los comedores escolares tienen problemas de personal, dificultades con la administración de los recursos y con las propias diluciones generadas por los mismos prestadores. En los comedores comunitarios no pocas veces se conforman estructuras clientelares y de poder dentro de la propia comunidad, desarticuladas de las necesidades y prioridades biológicas.

En general, estos programas alimentarios no están integrados a verdaderos criterios nutricionales, con sentido de oportunidad de las intervenciones, que prioricen, por ejemplo, los primeros 1.000 días desde la concepción, como momento crítico en el que se determina la epigenética de las enfermedades crónicas no transmisibles del adulto $^{(41)}$, y se utilicen alimentos de alta densidad nutricional y se provean, con sentido de regularidad, sostenibilidad y amplia cobertura para la población vulnerable ${ }^{(42)}$, aquellos alimentos y nutrientes de mayor valor biológico y más difíciles de adquirir por las familias.

\section{REFLEXIÓN FINAL}

La conceptualización de los programas alimentarios a partir de los tres componentes fundamentales de la seguridad alimentaria -disponibilidad, accesibilidad y utilización,facilita su comprensión para responder a distintos aspectos de los derechos y necesidades de alimentación y nutrición de la población. En este sentido, los programas actuales pueden ser reagrupados como se lo señala en la Tabla 3.

La aplicación de transferencias monetarias a través de cuentas individuales genera agilidad en los flujos presupuestarios, mayor 
Tabla 3. Tipología de los programas alimentarios desde los componentes de la seguridad alimentaria. Argentina, 1950-2015.

\begin{tabular}{lll} 
Componente & Función & Programa/Intervención \\
\hline Disponibilidad & $\begin{array}{l}\text { Apoyo a la producción } \\
\text { de alimentos }\end{array}$ & ProHuerta \\
& $\begin{array}{l}\text { Reintegro del impuesto } \\
\text { al valor agregado }\end{array}$ & Ley 27253 \\
\cline { 2 - 3 } Accesibilidad & $\begin{array}{l}\text { Subsidios al precio de } \\
\text { los alimentos }\end{array}$ & $\begin{array}{l}\text { Asignación Universal por Hijo (AUH) } \\
\text { Asignación por Embarazo (AE) } \\
\text { Precios Cuidados }\end{array}$ \\
\cline { 2 - 3 } & $\begin{array}{l}\text { Entrega de alimentos } \\
\text { Programa Materno Infantil (PMI) } \\
\text { Programa de Promoción Social Nutricional (PROSONU) } \\
\text { Programa Fondo Participativo de Inversión Social (FOPAR) }\end{array}$ \\
\hline Utilización & $\begin{array}{l}\text { Fortificación de } \\
\text { alimentos }\end{array}$ & $\begin{array}{l}\text { Ley 17259 (yodo en sal) } \\
\text { Ley 25459 (hierro y zinc en leche) } \\
\text { Ley 25630 (hierro y folatos en harina) }\end{array}$ \\
\cline { 2 - 3 } & $\begin{array}{l}\text { Apoyo a la utilización de } \\
\text { alimentos }\end{array}$ & $\begin{array}{l}\text { Plan Nacional de Seguridad Alimentaria (PNSA) } \\
\text { Fuente: Elaboración propia. }\end{array}$ \\
\hline
\end{tabular}

transparencia del uso de los recursos y más autonomía para los titulares de esos derechos en la elección de las compras. Es obvio que estas intervenciones no deben solo limitarse a simples transferencias sino que deben ir acompañadas de un programa educativo, campañas en los medios masivos y en la vía pública para promover consumos saludables.

El reintegro del impuesto al valor agregado (IVA) de los alimentos de la canasta básica -anunciado por el presidente Mauricio Macri en su discurso ante la Asamblea Legislativa, que entró en vigor en junio de 2016 con la promulgación de la Ley 27253- es una muy buena propuesta en la medida que se aplique selectivamente a los alimentos que aporten mayor densidad de nutrientes ${ }^{(43)}$.

Para categorizar los programas alimentarios dentro del esquema de la seguridad alimentaria, es justo mencionar aquellos que responden a la fortificación de alimentos. Estos están representados por el agregado de yodo como yoduro de potasio a la sal de consumo humano y animal, por la Ley 17259 promulgada en 1967; de hierro y zinc en la leche entera en polvo que se distribuye en los programas sociales, por la Ley 25459 promulgada en 2001; y de hierro y folatos en las harinas de trigo, por la Ley 25630 promulgada en 2003. Sobre esta última Ley, existe un proyecto de modificación del Art. $3^{\circ}$ (proyecto 3846-D-2014) para incorporar a las harinas de maíz y mandioca ${ }^{(44)}$.

No se conocen informes oficiales que den cuenta del monitoreo de los niveles de fortificación de yodo, hierro y folatos en sal, leche en polvo y harina, respectivamente. La Federación Argentina de Sociedades de Endocrinología (FASEM) realizó en todo el país, entre 1999 y 2008, un triple relevamiento con relación a la situación nutricional respecto del yodo: prevalencia de bocio en escolares, niveles urinarios de yodo y niveles de fortificación con yodo de diferentes marcas de las sales de consumo en los hogares ${ }^{(45)}$. Estos últimos estudios señalan alarmantes bajos niveles de fortificación ${ }^{(46)}$.

Por último, los programas orientados a mejorar la utilización de los alimentos se apoyan, fundamentalmente, en educación alimentaria y consejería, pero no hay intervenciones en el ambiente que condiciona 
las decisiones de hábitos de la población. En este sentido, la publicidad en general, y en particular la dirigida a niños, genera un gran condicionamiento de las conductas ${ }^{(47)}$.

Son muy escasas en Argentina las publicaciones abocadas a la evaluación de programas alimentarios, y tan solo una ha sido lo suficientemente amplia e integral como para mencionarla: se trata de la evaluación de comedores escolares realizada en 1985 por el Centro Interamericano para el Desarrollo Social de la Organización de los Estados Americanos (CIDES/OEA) ${ }^{(48)}$. Esta escasez contrasta con la multiplicidad de estudios que se pueden observar en Chile, por citar un país cercano. Sin evaluaciones de las intervenciones del Estado es, prácticamente, como transitar un camino a ciegas, con pocas posibilidades de mejorar su gestión y cumplir con sus objetivos y metas.

Una revisión general de los programas alimentarios requiere ser realizada desde su concepción, contenido y alcance, para dejar de ser meras ayudas sociales y transformarse en portadores del derecho a una mejor alimentación y nutrición.

\section{REFERENCIAS BIBLIOGRÁFICAS}

1. Harari YN. De animales a dioses. Buenos Aires: Debate; 2014.

2. Aguirre P. Alimentación humana, aspectos culturales: Las transiciones alimentarias en el tiempo de la especie. En: Braguinsky J, (comp). Obesidad, saberes y conflictos: Un tratado de obesidad. Buenos Aires: ACINDES, Editorial Médica AWWE; 2007. p. 207-237.

3. López X. La organización y consolidación del Estado nacional argentino en el tránsito al siglo XX: Los antecedentes de la política alimentaria en Argentina. En: Clemente A, (coord). Necesidades sociales y programas alimentarios: Las redes de la pobreza. Buenos Aires: Espacio Editorial; 2010. p. 79-89.

4. Comité de Seguridad Alimentaria Mundial. Acerca del CSA [Internet]. 2016 [citado 2 feb 2016]. Disponible en: https://goo.gl/ZOhrqK.

5. Foro de las ONG/OSC para la Soberanía Alimentaria. Soberanía alimentaria, un derecho para todos: Declaración política del Foro de las ONG/ OSC para la Soberanía Alimentaria. Roma: Foro de las ONG/OSC para la Soberanía Alimentaria; 2002.

6. Santarsiero LH. Las políticas sociales en el caso de la satisfacción de necesidades alimentarias: Algunos elementos conceptuales para su determinación. Trabajo y Sociedad. 2012;(18):159-176.
7. Pautassi L. Los difusos vínculos de articulación entre las políticas públicas y los derechos humanos. En: Vivero JL, Erazo X (ed). Derecho a la alimentación, políticas públicas e instituciones contra el hambre. Santiago: LOM Ediciones; 2009.

8. Herkovits D. La construcción de la malnutrición infantil: una etnografía sobre las condiciones y posibilidades que contribuyen a su producción y reproducción en hogares pobres de la Ciudad de Buenos Aires. Buenos Aires: CEDES-FLACSO; 2008.

9. Idiart A. Capacidades institucionales e implementación de programas sociales: las transformaciones de los programas Materno Infantiles en Argentina en la Última Década. Estado, Gobierno y Gestión Pública. 2007;(10):27-54.

10. Biernat C, Ramacciotti K. La tutela estatal de la madre y el niño en la Argentina: estructuras administrativas, legislación y cuadros técnicos (1936-1955). História, Ciências, Saúde-Manguinhos. 2008;15(2):331-351.

11. Britos S, O'Donnell A, Ugalde V, Clacheo R. Programas alimentarios en Argentina. Buenos Aires: CESNI; 2003.

12. Argentina. Salud Pública, Ley 20445: Autorízase la aplicación de un programa destinado a la protección de la salud de la madre y el niño [Internet]. 22 may 1973 [citado 12 mar 2016]. Disponible en: https://goo.gl/pqDbaJ. 
13. Instituto Nacional de Estadística y Censos. La pobreza en Argentina. Buenos Aires: INDEC; 1984.

14. Belmartino S. Crisis y reformulación de las políticas sociales. En: Suriano J, (dir). Dictadura y democracia: 1976-2001. Buenos Aires: Sudamericana; 2005. p. 225-280.

15. Grassi E. El asistencialismo en el estado neoliberal: la experiencia argentina de la década del 90. e-l@tina. 2003;1(4):25-48.

16. Argentina. Decreto 400/89: Bono Nacional Solidario de Emergencia [Internet]. 1 ago 1989 [citado 12 mar 2016]. Disponible en: https://goo.gl/nJFuby.

17. Rechazan un informe de Bauzá sobre los bonos. Diario La Prensa. 25 mar 1990.

18. Videla E. En busca del bono perdido. Página/12. 21 ene 1990

19. Golbert L. La asistencia alimentaria: un nuevo problema para los argentinos. En: Lumi S, Golbert L, Tenti Fanfani E. La mano izquierda del Estado. Buenos Aires: Miño y Dávila, CIEPP; 1992.

20. Verbitsky H. Robo para la corona: Los frutos prohibidos del árbol de la corrupción. Buenos Aires: Planeta; 1991.

21. Barcia H, Ivancich N. La carpa de Alí Babá: El grupo de los 8 contra la corrupción. Buenos Aires: Legasa; 1991.

22. Centro de Estudios para el Desarrollo Institucional, Fundación Gobierno y Sociedad, Fundación Grupo Sophia. Transferencia de recursos para programas alimentarios en las provincias: un análisis de lo sucedido en los años '90 (Documento 54) [Internet]. 2001 [citado 12 mar 2016]. Disponible en: https://goo.gl/f16llW.

23. Britos S. Programas alimentarios innovadores: Evaluación de una experiencia en el uso de tarjetas alimentarias [Internet]. Buenos Aires: Centro de Estudios Sobre Nutrición Infantil; 2005 [citado 12 mar 2016]. Disponible en: http://goo.gl/RDX85w.

24. Giraldez RC, Ruiz VA. Evaluando por productos: Decisiones y acciones en la búsqueda de un programa social más eficiente. En: Premio Anual ADEBA 1997. La eficiencia del gasto social. Buenos Aires: Mecanografix Editores; 1997. p. 65-118.

25. Presidencia de la Nación, Consejo Nacional de Coordinación de Políticas Sociales. Foro para un Plan Nacional de Alimentación y Nutrición. Buenos Aires: Consejo Nacional de Coordinación de Políticas Sociales; 2002.
26. Argentina. Ley 25724: Programa Nacional de Nutrición y Alimentación. [Internet]. 27 dic 2002 [citado 12 mar 2016]. Disponible en: https://goo. $\mathrm{gl} / \mathrm{T} 5 y f J \mathrm{~L}$.

27. Argentina, Ministerio de Desarrollo Social. Resolución 2040/03 [Internet]. 2003 [citado 12 mar 2016]. Disponible en: https://goo.gl/RMxU7W.

28. Administración Nacional de Seguridad Social. Asignación Universal por Hijo [Internet]. c2016 [citado 12 mar 2016]. Disponible en: http://goo. gl/9VHPbj.

29. Kliksberg B, Novacovsky I. El gran desafío: romper la trampa de la desigualdad desde la infancia, aprendizajes de la Asignación Universal por Hijo. Buenos Aires: Biblos; 2015. p. 312.

30. Ministerio de Salud. Encuesta Nacional de Nutrición y Salud [Internet]. Buenos Aires: Ministerio de Salud; 2007 [citado 12 mar 2016]. Disponible en: http://goo.gl/M9uPSi.

31. Ministerio de Salud, Dirección Nacional de Maternidad e Infancia. Situación de la lactancia materna en Argentina: Año 2011. Buenos Aires: Ministerio de Salud; 2011.

32. Ministerio de Salud. La alimentación de los niños menores de dos años. Buenos Aires: Ministerio de Salud; 2011.

33. Núñez P. Investigación sobre resultados del Plan Nacer/Programa Sumar [Internet]. Buenos Aires: Ministerio de Salud; 2015 [citado 10 mar 2016]. Disponible en http://goo.gl/57CmqV.

34. Ministerio de Salud. Encuesta Mundial de Salud Escolar. Buenos Aires: Ministerio de Salud, 2012. Disponible en http://goo.gl/j0O4As.

35. Ministerio de Salud, Instituto Nacional de Estadística y Censos. Tercera Encuesta Nacional de Factores de Riesgo para Enfermedades no Transmisibles [Internet]. Buenos Aires: Ministerio de Salud, INDEC; 2013 [citado 12 mar 2016]. Disponible en: http://goo.gl/ttjQu7.

36. World Health Organization. Report of the Comission on Ending Childhood Obesity [Internet]. Geneva: WHO; 2016 [citado 12 mar 2016]. Disponible en: http://goo.gl/VgAOHx.

37. Díaz Córdoba D. Consumo alimentario. En: Aguirre P, Díaz Córdoba D, Polischer G. Cocinar y comer en Argentina hoy. Buenos Aires: FUNDASAP; 2015. p. 78-106.

38. Maceira D, Stechina M. Intervenciones de política alimentaria en 25 años de democracia 
en Argentina. Revista Cubana de Salud Pública. 2011;37(1):44-60.

39. Nirenberg O, Brawerman J, Ruiz V. Evaluar para la transformación: Innovaciones en la evaluación de programas y proyectos sociales. Buenos Aires: Paidós; 2000.

40. lannotti L. Milk and dairy programmes affecting nutrition. En: Muehlhoff E, Bennett A, McMahon D, (ed). Milk and dairy products in human nutrition. Roma: FAO; 2013. p. 275-312.

41. Hanson MA, Gluckman PD. Early developmental conditioning of later health and disease: physiology or pathophysiology? Physiological Reviews. 2014;94:1027-1076.

42. Díaz A. Políticas alimentarias: algo más que programas compensatorios. Ponencia presentada en "De la universidad pública a la sociedad argentina: El Plan Fénix en vísperas del segundo centenario, una estrategia nacional de desarrollo con equidad". Buenos Aires: Facultad de Ciencias Económicas, Universidad de Buenos Aires; 2005.

43. Casa Rosada, Presidencia de la Nación. Palabras del presidente Mauricio Macri en la $134^{\circ}$ apertura de sesiones ordinarias del Congreso [Internet]. 1 mar 2016 [citado 12 mar 2016]. Disponible en: https://goo.gl/WgtGQ3.

44. Argentina. Proyecto de Ley 3846-D-2014: Anemias y malformaciones del tubo neural - Ley 25630: modificación del artículo 3, sobre incorporación de nutrientes a la harina de trigo de consumo humano [Internet]. 21 may 2014 [citado 12 mar 2016]. Disponible en: https://goo.gl/EJvL2l.

45. Pretell E, Niepomniszcze H. lodine deficiency persists in northern Argentina. IDD Newsletter. 2009;31(1):2-5.

46. López Linares S, Martín Heer I. Contenido de yodo en sal a nivel de puestos de venta provenientes de distintas localidades en tres regiones argentinas. Revista Argentina de Endocrinología y Metabolismo. 2014;51(2):59-65.

47. Britos S, Saraví A, Vilella F. Alimentación saludable en la Argentina: Logros y desafíos. Buenos Aires: Orientación Gráfica Editora; 2013.

48. Organización Panamericana de la Salud. Evaluación de un programa de alimentación escolar: el caso argentino. Buenos Aires: OPS; 1990.

\section{FORMA DE CITAR}

Abeyá Gilardon EO. Una evaluación crítica de los programas alimentarios en Argentina. Salud Colectiva. 2016;12(4):589604. doi: 10.18294/sc.2016.935

Recibido: 6 de abril de 2016 | Versión final: 2 de julio de 2016 | Aprobado: 9 de agosto de 2016

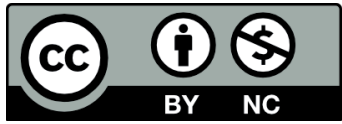

Este obra está bajo una licencia de Creative Commons Reconocimiento-NoComercial 4.0 Internacional. Reconocimiento - Permite copiar, distribuir y comunicar públicamente la obra. A cambio, se debe reconocer y citar al autor original. No Comercial - Esta obra no puede ser utilizada con finalidades comerciales, a menos que se obtenga el permiso. 